\title{
First report of dollar spot of Agrostis stolonifera, Poa pratensis, Festuca arundinacea and Zoysia japonica caused by Sclerotinia homoeocarpa in China
}

\author{
C.C. LV ${ }^{1}$, T. Hsiang ${ }^{2}$, J.Q. Li ${ }^{1}$ and L.X. Luo ${ }^{1 *}$ \\ ${ }^{1}$ Department of Plant Pathology, China Agricultural University/Key Laboratory of Plant Pathology, Ministry of Agriculture, \\ Beijing 100193, China; ${ }^{2}$ Environmental Sciences, University of Guelph, Guelph, Ontario, N1G 2 W1, Canada \\ *E-mail: luolaixin@cau.edu.cn
}

Received: 25 Apr 2011. Published: 30 Jun 2011. Keywords: turfgrass, fungal plant disease

Sclerotinia homoeocarpa causes dollar spot disease on many warm and cool season grasses. In Asia, this disease has only been formally reported in Japan (Hsiang \& Mahuku, 1999) and south China (Lv et al., 2010). In China, the first anecdotal reports of dollar spot disease on turf were from the late 1990s, mainly in south China. For the past few years, this disease has been observed on cool season turfgrasses in north China with increasing severity. Outbreaks at golf courses in Beijing were observed in 2008, 2009 and 2010, where disease was first seen in early spring and developed from late spring until temperatures exceeded $32^{\circ} \mathrm{C}$ in early summer. When temperatures dropped below this in late summer, the disease flared up again until mid-autumn. Maximum disease development usually occurred on turf deficient in nitrogen and water, especially with warm days and cool nights with heavy dew.Characteristic hourglass foliar lesions were at first chlorotic, and then appeared water-soaked and finally bleached, with typical reddish-brown borders. Small patches appeared on turfgrasses, with a typical incidence of patches of $1-5 / \mathrm{m}^{2}$ and up to $50 / \mathrm{m}^{2}$ under high disease levels.

In late May 2009, disease samples were collected from several golf courses in Beijing, and fungal isolates obtained from creeping bentgrass (Agrostis stolonifera), Kentucky bluegrass (Poa pratensis), tall fescue (Festuca arundinacea) and zoysia grass (Zoysia japonica). On potato dextrose agar (PDA) medium, white fluffy mycelia covered the whole petri dish three days after incubation, and formed brown columnar structures in the centres by seven days. Cultures more than 14 days old became cinnamon coloured and formed dark brown or black substratal stroma. Four-week-old seedlings of A. stolonifera cv. T-1, P. pratensis cv. Nu-Glade, F. arundinacea cv. Arid 3 and Z. japonica cv. Lanyin No. 3 were inoculated with 5-mm-diameter mycelial plugs of respective isolates of the fungus from five-day-old PDA cultures, by placing plugs onto the leaves and incubating under humid conditions. White aerial hyphae and leaf lesions were observed within seven days after inoculation on all four species of turfgrass, but no disease was seen with sterile agar plugs as inoculum. The fungus was re-isolated from lesions to satisfy Koch's postulates. DNA from one isolate per host species was amplified with internal transcribed spacer (ITS) primers and sequenced to yield $610 \mathrm{bp}$ fragments (GenBank Accession Nos. GQ924923-GQ924926). Sequences were analysed by BLAST against GenBank, and showed over $99 \%$ identity with ITS regions of sequences identified as S. homoeocarpa. To the best of our knowledge, this is the first formal report of dollar spot disease on creeping bentgrass, Kentucky bluegrass, tall fescue or zoysia grass in China.

\section{References}

Hsiang T, Mahuku GS, 1999. Genetic variation within and between southern Ontario populations of Sclerotinia homoeocarpa. Plant Pathology 48, 83-94. [doi:10.1046/j.1365-3059.1999.00306.x]

Lv CC, Luo LX, Li JQ, Hsiang T, 2010. First report of dollar spot of seashore paspalum (Paspalum vaginatum) caused by Sclerotinia homoeocarpa in South China. Plant Disease 94, 373.

[doi:10.1094/PDIS-94-3-0373B]

To cite this report: Lv CC, Hsiang T, Li JQ, Luo LX, 2011. First report of dollar spot of Agrostis stolonifera, Poa pratensis, Festuca arundinacea and Zoysia japonica caused by Sclerotinia homoeocarpa in China. New Disease Reports 23, 37. 